method S2

\title{
Construction of pET28aAlacI
}

pET28a was amplified with primers pET28aAlacIs (5'-GCCCAGTAGTAGGTTGAGGC-3') and pET28a_lacla (GAAGATCT ${ }^{\text {BglII }}$ TCGGTTTCCGTGTTTCGTA). The PCR product was gel-purified and digested with BgIII, and self-ligated (one BglII site existed on pET28a, the other was introduced by primer pET28a $\Delta$ lacIa). In this way we deleted the lacI from pET28a and made pET28aAlacI. Ligation mixtures were transformed into E. coli DH5a chemical component cells, and the resulting transformants were plated on LB agar with kanamycin. 